\title{
Nominata de Avaliadores
}

Agradecemos a todos os avaliadores ad hoc, listados a seguir, que estiveram à disposição da Revista Research, Society and Development durante o ano de 2017:

\begin{tabular}{|c|c|c|}
\hline Nome & Instituição & País \\
\hline Acsa Nara de Araújo Brito Barros & Universidade Federal do Rio Grande do Norte & Brasil \\
\hline Adriam Camacho Domínguez & Universidad Laica Vicente Rocafuerte de Guayaquil & Equador \\
\hline Adriane Cenci & Universidade Federal do Rio Grande do Norte & Brasil \\
\hline Alex Paubel Junger & Universidade Federal do $\mathrm{ABC}$ & Brasil \\
\hline Amanda Silva Alves & Universidade Federal Fluminense & Brasil \\
\hline Ana Acácia Araújo de Souza Eda & Serviço Nacional de Aprendizagem Comercial & Brasil \\
\hline André Luís Rossoni Ferreira & Fundação Comunitária de Ensino Superior de Itabira & Brasil \\
\hline André Luiz Thomaz de Souza & Faculdades Integradas do Vale do Ribeira & Brasil \\
\hline Andrea Cristina Marin Schmidt & Centro Paula Souza & Brasil \\
\hline Andrialex William da Silva & Universidade Federal do Rio Grande do Norte & Brasil \\
\hline Anna Gabriella de Souza Cordeiro & Universidade Federal do Rio Grande do Norte & Brasil \\
\hline Bárbara de Oliveira Prado Sousa & Universidade de São Paulo & Brasil \\
\hline Bruno Carpinetti & Universidad Nacional Arturo Jauretche & Argentina \\
\hline Carlos Adriano Martins & Universidade Cidade de São Paulo & Brasil \\
\hline Carlos Anibal Peris Castiglioni & Universidad Nacional de Asunción & Paraguai \\
\hline Carlos Eymel Campos Rodriguez & Universidade Federal de Itajubá & Brasil \\
\hline Carmencitta Ignatti & Faculdade Peruíbe & Brasil \\
\hline Carmino Hayashi & Universidade Federal do Triângulo Mineiro & Brasil \\
\hline Celina Maria De Souza Olivindo & Universidade Federal do Piauí & Brasil \\
\hline Cláudio Boghi & Universidade Anhembi Morumbi & Brasil \\
\hline Claudio Cleverson de Lima & Universidade Feevale & Brasil \\
\hline Clegiane Santos Bezerra Dantas & Secretaria de Educação e Cultura do RN & Brasil \\
\hline Cristiane Alves Dantas & Universidade Federal do Rio Grande do Norte & Brasil \\
\hline Dário Moreira Pinto Junior & Centro Universitário de Barra Mansa & Brasil \\
\hline Desiré Nguessan & Universidade de Mogi das Cruzes & Brasil \\
\hline Dorlivete Moreira Shitsuka & Universidade Federal de Itajubá & Brasil \\
\hline Ednete Morais Costa Elias & Serviço Brasileiro de Apoio às Micro e Pequenas Empresas & Brasil \\
\hline Elisabete Amadeu Montero & Universidade Católica de Santos & Brasil \\
\hline Eniel do Espírito Santo & Universidad de la Empresa & Uruguai \\
\hline Enir da Silva Fonseca & Universidade de Ribeirão Preto & Brasil \\
\hline Érico Gurgel Amorim & Universidade Federal do Rio Grande do Norte & Brasil \\
\hline Eugenio Gustavo Coconier & Universidad de Buenos Aires & Argentina \\
\hline Fabiano Cunha Marinho & Centro Universitário de Barra Mansa & Brasil \\
\hline Facundo Nazareno Saxe & Universidad Nacional de La Plata & Argentina \\
\hline Felix Raúl España Cuellar & Universidad Mayor de San Andres & Bolívia \\
\hline Fernanda Abreu de Moraes Figueiredo & Centro Fed.de Ed. Tecnológica Celso Suckow da Fonseca & Brasil \\
\hline Freddy Oropeza & Universidad Pedagógica Experimental Libertador & Venezuela \\
\hline Gessica Fabiely Fonseca & Universidade Federal do Rio Grande do Norte & Brasil \\
\hline Gillyane Dantas dos Santos & Universidade Federal do Rio Grande do Norte & Brasil \\
\hline Giovanna Moura Calazans & Universidade Federal de Minas Gerais & Brasil \\
\hline Gleicemares Maria Marques & Universidade Federal de Itajubá & Brasil \\
\hline
\end{tabular}


Research, Society and Development, v. 6, n. 4, p. 382-384, dez. 2017

\begin{tabular}{|c|c|c|}
\hline Nome & Instituição & País \\
\hline Gleice Virginia Medeiros de Azambuja Elali & Universidade Federal do Rio Grande do Norte & Brasil \\
\hline Graziele Lage Alves Santiago & Fundação Comunitária de Ensino Superior de Itabira & Brasil \\
\hline Hector José Garcia Mendoza & Universidade Federal de Roraima & Brasil \\
\hline Henry Anibal Angulo Serrano & Universidad Católica Boliviana San Pablo & Bolívia \\
\hline Ilane Ferreira Cavalcante & Instituto Fed. de Ed., Ciência e Tecnologia do Federal do RN & Brasil \\
\hline Isabela Cristina Santos de Morais & Universidade Federal do Rio Grande do Norte & Brasil \\
\hline Ivair Fernandes de Amorim & Instituto Federal de São Paulo & Brasil \\
\hline Jael Glauce Fonseca & Universidade Federal da Bahia & Brasil \\
\hline Jander Temístocles de Oliveira & Faculdade de Ciências Médicas da Santa Casa de São Paulo & Brasil \\
\hline Jaqueline Costa Castilho Moreira & Universidade Estadual Paulista Júlio de Mesquita Filho & Brasil \\
\hline Joelma Moraes Silva & Universidad de la Empresa & Uruguai \\
\hline José Blas Villalba Giménez & Universidad del Cono Sur de las Américas & Paraguai \\
\hline Josefa da Conceição Silva & Universidade Federal de Roraima & Brasil \\
\hline Josirene de Carvalho Barbosa & Instituto Fed. de Educação, Ciência e Tecnologia - Sul de MG & Brasil \\
\hline Juni Silveira Cordeiro & Fundação Comunitária de Ensino Superior de Itabira & Brasil \\
\hline Kelly Danielly da Silva Alcantara Fratta & Universidade Federal do ABC & Brasil \\
\hline Konstantinos Ravanis & University of Patras & Grécia \\
\hline Larissa Scandelari Ferreira & Claretiano - Centro Universitário de Batatais & Brasil \\
\hline Leila Zardo Puga & Pontifícia Universidade Católica de São Paulo & Brasil \\
\hline Lenina Lopes Soares Silva & Instituto Fed. de Ed., Ciência e Tecnologia do Federal do RN & Brasil \\
\hline Loan José Landaeta & Universidad Pedagógica Experimental Libertador & Venezuela \\
\hline Luis Camilo Kunstek Salinas & Universidad Católica Boliviana San Pablo & Bolívia \\
\hline Luís Felipe Pissaia & Universidade do Vale do Taquari & Brasil \\
\hline Luís Henrique Borges & Universidade de Uberaba & Brasil \\
\hline Marcelo Dias Pereira & Centro Universitário da FEI & Brasil \\
\hline Márcia Torres Neri Soares & Universidade do Estado da Bahia & Brasil \\
\hline Maria Carolina de Abreu & Universidade Federal do Piauí & Brasil \\
\hline Mariane de Araújo Soares & Universidade Federal do Rio Grande do Norte & Brasil \\
\hline Marina Gonçalves Gregorutti & Claretiano - Centro Universitário de Batatais & Brasil \\
\hline Mariza Suely Assis Meireles & Universidade Federal de Itajubá & Brasil \\
\hline Max Leandro de Araújo Brito & Faculdade Natalense de Ensino e Cultura & Brasil \\
\hline Maximiliano Bezada Díaz & Universidad Pedagógica Experimental Libertador & Venezuela \\
\hline Mayara Mirela Balbino de Souza & Universidade Federal do $\mathrm{ABC}$ & Brasil \\
\hline Nanci Neves Cardim & Universidade Federal Fluminense & Brasil \\
\hline Nayane da Silva Abrahão & Claretiano - Centro Universitário de Batatais & Brasil \\
\hline Olivia Morais de Medeiros Neta & Universidade Federal do Rio Grande do Norte & Brasil \\
\hline Orivaldo de Lira Tavares & Universidade Federal do Espírito Santo & Brasil \\
\hline Orlando José González Clemente & Universidad Pedagógica Experimental Libertador & Venezuela \\
\hline Pablo Lopes Quintão & Fundação Comunitária de Ensino Superior de Itabira & Brasil \\
\hline Paulo César Pontes Fraga & Universidade Federal de Juiz de Fora & Brasil \\
\hline Paulo Cruz Correia & Universidade Estadual do Paraná & Brasil \\
\hline Paulo Henrique da Silva & Universidade Federal do Piaú & Brasil \\
\hline Priscila Bernardo Martins & Universidade Cidade de São Paulo & Brasil \\
\hline Priscilla Chantal Duarte Silva & Universidade Federal de Itajubá & Brasil \\
\hline Rafael Balbino Cardoso & Universidade Federal de Itajubá & Brasil \\
\hline Raquel Fernandes de Macedo & Universidade Federal do $\mathrm{ABC}$ & Brasil \\
\hline Renata Andrea Fernandes Fantacini & Claretiano - Centro Universitário de Batatais & Brasil \\
\hline Renata Caroline Costa de Freitas & Centro de Ensino Profissionalizante do RN & Brasil \\
\hline Rhodiney Vaz Martins & Universidade Virtual do Estado de São Paulo & Brasil \\
\hline Ricardo Luiz Perez Teixeira & Universidade Federal de Itajubá & Brasil \\
\hline Rodrigo Leonardo Guillén Bretón & Universidad del Caribe & Rep. Dominicana \\
\hline Ronara Cristina Bozi dos Reis & Universidade Federal de Itajubá & Brasil \\
\hline
\end{tabular}




\begin{tabular}{||l|l|c|}
\hline \multicolumn{1}{|c|}{ Nome } & \multicolumn{1}{c|}{ Instituição } & País \\
\hline Rosa Maria da Motta Azambuja & Universidade Católica do Salvador & Brasil \\
\hline Rosângela Martins Carrara & Universidad Autônoma de Madrid & Espanha \\
\hline Ruan Kléberson Pereira da Silva & Universidade Federal do Rio Grande do Norte & Brasil \\
\hline Sergio Foghin Pillin & Universidad Pedagógica Experimental Libertador & Brasil \\
\hline Tabita Aija Silva Moreira & Universidade Estácio de Sá & Brasil \\
\hline Valdilene Gonçalves Machado Silva & Universidade do Estado de Minas Gerais & Bolívia \\
\hline Víctor Hugo Mamani Yapura & Universidad Indígena Quechua Casimiro Huanca & Brasil \\
\hline Viviane Sartori & Universidade Federal de Santa Catarina & Espanha \\
\hline Weimar Giovanni Iño Daza & Universidad de Jaume I & Brasil \\
\hline Willyana Freire Bispo & Centro de Ensino Profissionalizante do RN & Brasil \\
\hline Ykaro Richard Oliveira & Universidade Federal do Piaúi & \\
\hline
\end{tabular}

Dr. Ricardo Shitsuka

Editor 